\title{
Spatiotemporal amplitude and phase retrieval of space-time coupled ultrashort pulses using the Shackled-FROG technique
}

\author{
Eleonora Rubino, ${ }^{1,5}$ Daniele Faccio, ${ }^{1,2, *}$ Luca Tartara, ${ }^{3,5}$ Philip K. Bates, ${ }^{2}$ Olivier Chalus, ${ }^{2}$ \\ Matteo Clerici, ${ }^{1,5}$ Fabio Bonaretti, ${ }^{1}$ Jens Biegert, ${ }^{2,4}$ and Paolo Di Trapani ${ }^{1,5}$ \\ ${ }^{1}$ CNISM and Department of Physics and Mathematics, University of Insubria, Via Valleggio 11, IT-22100 Como, Italy \\ ${ }^{2}$ ICFO-Institut de Ciènces Fotòniques, Mediterranean Technology Park, 08860 Castelldefels, Barcelona, Spain \\ ${ }^{3}$ Department of Electronics, University of Pavia, Via Ferrata 1, I-27100 Pavia, Italy \\ ${ }^{4}$ ICREA Institució Catalana de Recerca i Estudis Avançats, 08010 Barcelona, Spain \\ ${ }^{5}$ Virtual Institute for Nonlinear Optics, Centro di Cultura Scientifica Alessandro Volta, Villa Olmo, \\ Via Simone Cantoni 1, 22100 Como, Italy \\ *Corresponding author: daniele.faccio@uninsubria.it
}

\begin{abstract}
We demonstrate the validity of the Shackled-frequency-resolved-optical-gating technique for the complete characterization, both in space and in time, of ultrashort optical pulses that present strong angular dispersion. Combining a simple imaging grating with a Hartmann-Shack sensor and standard frequency-resolvedoptical-gating detection at a single spatial position, we are able to retrieve the full spatiotemporal structure of a tilted pulse.
\end{abstract}

Several techniques have been proposed for retrieving the amplitude and phase profile of an ultrashort pulse, with most methods allowing the reconstruction of the electric field either in the time or in the space domain. The main assumption lying behind these techniques is that the spatial and temporal features of the pulse are independent. The most known examples are frequency-resolved optical gating (FROG) [1] and spectral interferometry for direct electric-field reconstruction (SPIDER) [2], which allow for the retrieval of the temporal phase. On the other hand, spatial wavefront reconstruction is made possible by means of interferometric techniques or by a Hartmann-Shack (H-S) sensor [3] to obtain spatial information about monochromatic pulses.

However, in many cases, the need for the characterization of complex wave packets featured by strong space-time coupling arises. More refined techniques have, thus, been developed that are able to provide the complete reconstruction of the electric field (E-field) of the pulse as a function of both space and time simultaneously. Many of these approaches basically originate from an extension of the FROG and SPIDER techniques to the spatial coordinate, such as spatial encoded arrangement for temporal analysis by dispersing a pair of light E-fields (SEA TADPOLE) $[4,5]$, spatially and temporally resolved intensity and phase evaluation device: full information from a single hologram (STRIPED FISH) [6], spatial encoded arrangement SPIDER (SEA SPIDER) [7], and complete retrieval of the optical amplitude and phase using the $\left(k_{\perp}, \omega\right)$ spectrum (CROAK) [8].

The combination of $\mathrm{H}-\mathrm{S}$ wavefront reconstruction with temporal autocorrelation was first demonstrated by Grunwald et al. [9] and the possibility to extend this concept to include full phase retrieval with a FROG measurement was also suggested. A similar combination of separately retrieved temporal and spatial phase profiles was also studied by Rivet [10]. However, the method suggested in [9] implies a FROG measurement at each lenslet spatial position in the $\mathrm{H}-\mathrm{S}$ array rendering it computationally rather tedious. Furthermore, no indications are given on how to combine the retrieved information in order to obtain the full spatiotemporal amplitude and phase profile of the laser pulse. Recently, these difficulties have been solved with the experimental demonstration of the Shackled-FROG technique [11]. This is based on a single temporal FROG measurement combined with the spatial amplitude and phase obtained with a single H-S measurement. In [11] the technique was applied to a space-time coupled pulse, the Bessel-X pulse, in which angular dispersion is negligible. This allowed us to simplify the spatial profile acquisition, under the assumption that the spatial phase was the same for each monochromatic component of the field.

In this Letter, we further implement this technique as an innovative and handy way to completely characterize the field of complex ultrashort pulses characterized by space-time coupling and angular dispersion, i.e., in which the spatial phase varies with the temporal spectrum. This is obtained by using a $\mathrm{H}-\mathrm{S}$ sensor placed in the imaging plane of a spectrometer in order to characterize the amplitude and phase of the spatially-resolved spectrum. The validity of this technique is demonstrated by characterizing the prototype of the space-time coupled pulses, i.e., a tilted pulse.

The experimental layout is shown in Fig. 1. The tilted pulse was generated by a $795 \mathrm{~nm}, 45 \mathrm{fs}, 4 \mathrm{~mm}$ FWHM diameter pulse, delivered at $5 \mathrm{kHz}$ repetition rate (KM Labs) and impinging on a 300 lines/mm dif- 


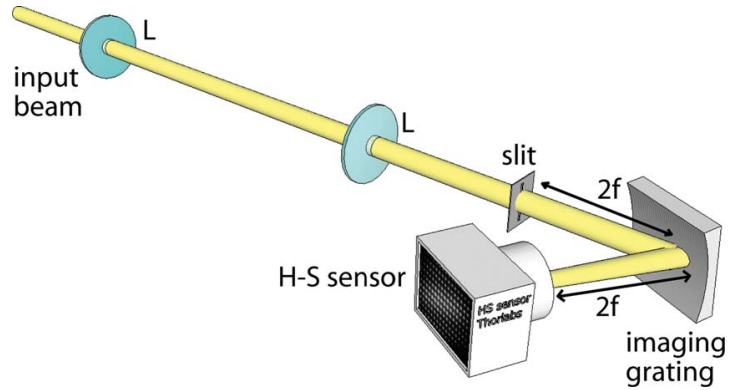

Fig. 1. (Color online) Experimental layout for the spatial diagnostics: $\mathrm{H}-\mathrm{S}$ sensor combined with an imaging concave grating for the retrieval of the phase of the $(r, \lambda)$ spectrum. The input slit of the imaging grating is placed in the image plane of the $4 f$ imaging system made by lenses $L$.

fraction grating, which induces angular dispersion resulting in a pulse front tilt of $18.3^{\circ}$. The tilted pulse is then imaged by a $4 f$ telescope (made by lenses $L$ in Fig. 1) onto the diagnostics, i.e., at the input slit of a spectrometer for the spatial measurement described below, or on the second harmonic crystal used for the FROG temporal measurement.

The spatial amplitude and phase profile is obtained using a commercial H-S sensor (WFS150C Thorlabs, $150 \mu \mathrm{m}$ lenslet pitch, $3.7 \mathrm{~mm}$ effective focal length). It is important to note that, due to the angular dispersion (i.e., the dependence of frequency on angle) of the tilted pulse, the spatial phase is different at each frequency implying that the $\mathrm{H}-\mathrm{S}$ measurement must be repeated at each wavelength of the laser pulse. To account for the angular dispersion of the tilted pulse, the H-S acquisition may be performed by a series of measurements using a set of interference filters as in [12]. In this way, the amplitude and phase information is obtained over the full two-dimensional transverse profile albeit at the expense of a number of repeated measurements and with a rather coarse wavelength resolution, limited by the available interference filters. Another option, that we choose here, is to place the $\mathrm{H}-\mathrm{S}$ sensor in the imaging plane of a spectrometer. The spectrometer is composed by a single imaging grating (concave blazed holographic grating, 430 lines/mm, Edmund Optics) that images the vertical slice of the input beam (selected by the input slit) onto the vertical axis of the output imaging plane and spreads the spectrum along the horizontal plane. The H-S sensor will thus reconstruct in a single shot the spatial phase at each wavelength with a high resolution. The downside of this approach is the loss of information along the horizontal axis of the input pulse. However, we note that this is perfectly acceptable if no spatial chirp is expected along the horizontal axis (as in the present case) or for pulses with cylindrical symmetry. We note that ideally the spectrometer should reproduce both the input amplitude and phase at the output imaging plane. This is usually achieved using an imaging spectrometer, i.e., a spectrometer in which the imaging is performed by a two-lens telescope. In the present case we are using a single imaging grating that may be considered as the equivalent of a planar grating combined with a focusing mirror and such a combination will not, in general, reproduce both amplitude and phase at the output. In other words, the imaging grating will contribute to the output phase profile. However, the $\mathrm{H}-\mathrm{S}$ sensor allows us to account for this contribution and subtract it from the final measurement by an independent calibration. This is performed by sending a plane wave onto the input slit obtained by a highly expanded He-Ne laser beam. The measured output phase in this case gives us directly the additive phase contribution from the imaging grating. This spatial phase contribution is therefore subtracted at each wavelength from the subsequent measurements.

In Fig. 2, we show the H-S acquisition of the $(r, \lambda)$ spectrum (a) together with the corresponding $(r, \lambda)$ intensity profile (b). The normalized phase of the $(r, \lambda)$ spectrum is depicted in Fig. 2(c) and may be compared to the theoretical one in Fig. 2(d) calculated for the grating used in the experiment.

Finally, once the spatial phases at each wavelength have been retrieved, these are linked together using a second-harmonic-generation FROG measurement at $r=0$, under the assumption that all frequencies within the pulse are present at this spatial location. In Fig. 3 we show the experimental (a) and the retrieved (b) FROG spectrograms. The temporal and spectral profile of the retrieved pulse are shown in Figs. 3(c) and 3(d), respectively.

By normalizing the $\mathrm{H}-\mathrm{S}(r, \lambda)$ measurements with the spectral intensity and phase profile retrieved with the FROG, the full spatiotemporal structure of the pulse is finally obtained. The space-time profile of the reconstructed pulse is shown in Fig. 4. In particular, Fig. 4(a) clearly presents the intensity distribution which is typical of a tilted pulse in the $(r, t)$ domain. From the slope of the line of maximum intensity we retrieved a tilt angle of $\sim 19.1^{\circ}$, in good agreement with the expected value. Figure $4(\mathrm{~b})$ shows the retrieved spatiotemporal phase of the tilted pulse which shows a clear curvature along both the spatial and the temporal coordinate.
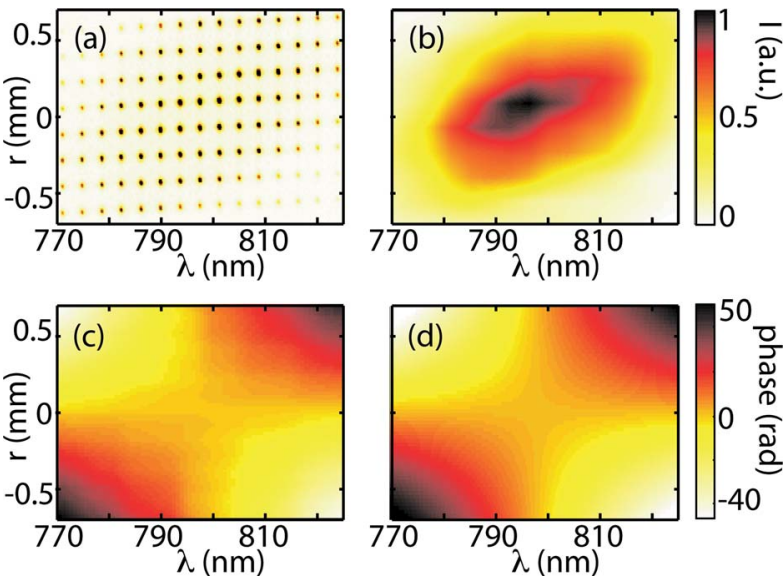

Fig. 2. (Color online) Spatially resolved spectrum measurements. (a) H-S acquisition and (b) corresponding normalized $(r, \lambda)$ intensity profile. (c) Retrieved phase normalized at $r=0$ with the spectral phase of Fig. 3(d). (d) Theoretical phase of the $(r, \lambda)$ spectrum for a tilted pulse with tilt angle equal to $18.3^{\circ}$. 

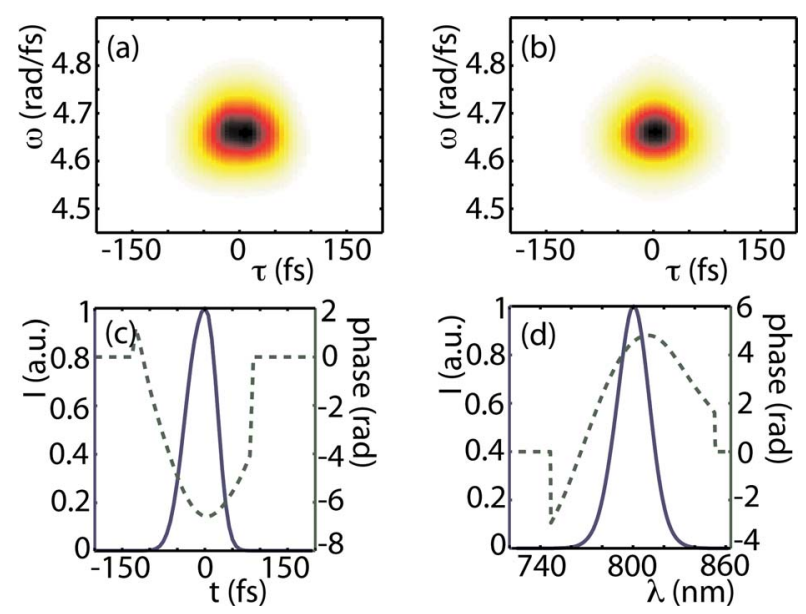

Fig. 3. (Color online) (a) Experimental spectrogram. (b) Last spectrogram retrieved by the iterative algorithm. (c) and (d) Retrieved temporal pulse and power spectrum, respectively: normalized intensity (blue solid line) and phase (green dashed line). The retrieved pulse duration is $\sim 63 \mathrm{fs}$ FWHM.

In conclusion, we demonstrate the validity of the innovative technique Shackled-FROG based on the combination of FROG, and $\mathrm{H}-\mathrm{S}(r, \lambda)$ spectrum measurements to completely characterize in space and time the amplitude and phase of an ultrashort space-
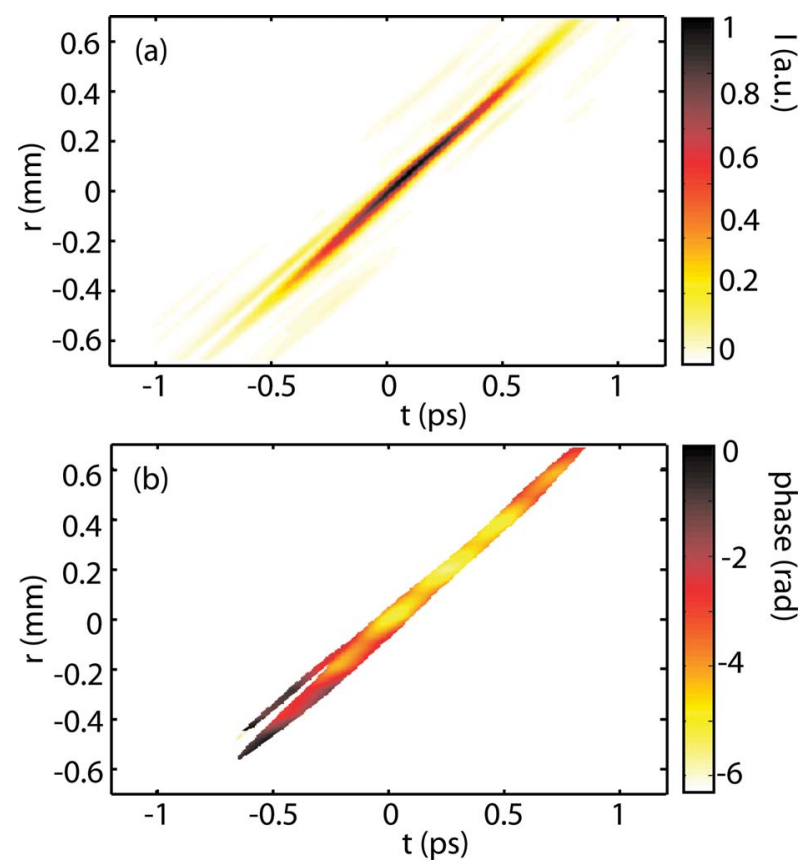

Fig. 4. (Color online) (a) Reconstructed near-field intensity and (b) phase profile of a tilted pulse in the space-time domain. time coupled pulse with strong angular dispersion. At the best of our knowledge this technique is the most simple and cost effective among the other diagnostics developed for fully characterizing the field of ultrashort laser pulses. Moreover, the employment of a compact, simple imaging grating in place of an expensive and often not available imaging spectrometer renders this technique even more profitable.

These results have been obtained in the context of the STELLA-2009 experimental school (http:// www.vino-stella.eu), held at ICFO in Castelldefels in the framework of the MC Chair project "STELLA" MEXC-CT-2005-025710. The authors acknowledge support from the Consorzio Nazionale Interuniversitario per le Scienze della Materia (CNISM), progetto INNESCO. D. Faccio acknowledges support from Marie Curie grant, contract PIEF-GA-2008220085. J. Biegert acknowledges support from the Spanish Ministry of Science and Innovation through its Consolider-Ingenio 2010 Program (grant SAUULCSD 2007-00013), "Plan Nacional" (grant FIS200806368-C02-01/02), as well as Agència de Gestió d'Ajuts Universitaris i de Recerca (AGAUR) through grant SGR 2009.

\section{References}

1. R. Trebino and D. J. Kane, J. Opt. Soc. Am. A 10, 1101 (1993).

2. C. Iaconis and I. A. Walmsley, IEEE J. Quantum Electron. 35, 501 (1999).

3. R. V. Shack and B. C. Platt, Opt. Science Cent. Newsl. 5, 15 (1971).

4. P. Bowlan, P. Gabolde, A. Schreenath, K. McGresham, and R. Trebino, Opt. Express 14, 11892 (2006).

5. P. Bowlan, P. Gabolde, and R. Trebino, Opt. Express 15, 10219 (2007).

6. P. Gabolde and R. Trebino, Opt. Express 14, 11460 (2006).

7. A. S. Wyatt, I. A. Walmsley, G. Stibenz, and G. Steinmeyer, Opt. Lett. 31, 1914 (2006).

8. F. Bragheri, D. Faccio, F. Bonaretti, A. Lotti, M. Clerici, O. Jedrkiewicz, C. Liberale, S. Henin, L. Tartara, V. Degiorgio, and P. Di Trapani, Opt. Lett. 33, 2952 (2008).

9. R. Grunwald, U. Neumann, U. Griebner, K. Reimann, G. Steinmeyer, and V. Kebbel, Opt. Lett. 28, 2399-2401 (2003).

10. S. Rivet, Ph.D. thesis (University of Bordeaux, 2001).

11. F. Bonaretti, D. Faccio, M. Clerici, J. Biegert, and P. Di Trapani, Opt. Express 17, 9804 (2009).

12. C. P. Hauri, J. Biegert, U. Keller, B. Schaefer, K. Mann, and G. Marowski, Opt. Lett. 30, 1563 (2005). 